\title{
Valve-sparing and valve-replacing techniques for aortic root replacement in patients with Marfan syndrome: Analysis of early outcome
}

Irina V. Volguina, PhD, ${ }^{a}$ D. Craig Miller, MD,${ }^{\mathrm{b}}$ Scott A. LeMaire, MD, ${ }^{\mathrm{a}}$ Laura C. Palmero, MPH, ${ }^{\mathrm{a}}$ Xing Li Wang, MD, PhD, ${ }^{\mathrm{a}}$ Heidi M. Connolly, MD, ${ }^{\mathrm{c}}$ Thoralf M. Sundt, III, MD, ${ }^{\mathrm{c}}$ Joseph E. Bavaria, MD, ${ }^{\mathrm{d}}$ Harry C. Dietz, MD, ${ }^{\mathrm{e}}$ Dianna M. Milewicz, MD, $\mathrm{PhD},{ }^{\mathrm{f}}$ and Joseph S. Coselli, MD, ${ }^{\mathrm{a}}$ on behalf of the Aortic Valve Operative Outcomes in Marfan Patients study group

This article is a corrected and republished version of the article that originally appeared in $J$ Thorac Cardiovasc Surg. 2009;137:641-9.

Objective: A prospective, international registry study was initiated to provide contemporary comparative data on short-term clinical outcomes after aortic valve-sparing and aortic valve-replacing root operations in patients with Marfan syndrome. The purpose of this initial report is to describe the study design and to compare early outcomes in the first 151 enrolled patients.

Methods: We assessed 30-day outcomes in 151 patients who met strict Ghent diagnostic criteria for Marfan syndrome and underwent aortic root replacement with either valve-replacing $(n=46)$ or valve-sparing techniques $(n=105)$ at one of 18 participating centers. In the valve replacement group, a mechanical composite valve graft was used in $39(85 \%)$ patients and a bioprosthetic valve in $7(15 \%)$. In the valve-sparing group, David V procedures were performed in $57(54 \%)$ patients, David I in $38(36 \%)$, David IV in $8(8 \%)$, Florida sleeve in 1 $(1 \%)$, and Yacoub remodeling in $1(1 \%)$.

Results: No in-hospital or 30-day deaths occurred. Despite longer crossclamp and cardiopulmonary bypass times in the valve-sparing group, there were no significant between-group differences in postoperative complications. Thirty-day valve-related complications occurred in $2(4 \%)$ patients undergoing valve replacement and in $3(3 \%)$ undergoing valve-sparing procedures $(P=.6)$.

Conclusions: The analysis of early outcomes revealed that valve-sparing techniques were the most common approach to root replacement in patients with Marfan syndrome in these centers. The complexity of valve-sparing root replacement did not translate into any demonstrable adverse early outcomes. Subsequent analysis will compare the 3-year durability of these two surgical approaches.

Publisher's note.-Due to a production error, the original article that printed in the March 2009 issue of The Journal of Thoracic and Cardiovascular Surgery $(2009 ; 137: 641-9)$ was not in its corrected and final form. The article is republished in its entirety below.

From Baylor College of Medicine and the Texas Heart Institute at St. Luke's Episcopal Hospital, Houston, Tex ${ }^{\mathrm{a}}$; Stanford University, Stanford, Calif ${ }^{\mathrm{b}}$; Mayo Clinic, Rochester, Minn ${ }^{\mathrm{c}}$; University of Pennsylvania, Philadelphia, $\mathrm{Pa}^{\mathrm{d}}$; Johns Hopkins Hospital, Baltimore, $\mathrm{Md}^{\mathrm{e}}$; and University of Texas Health Science Center, Houston, Tex. ${ }^{\mathrm{f}}$

Funded by the National Marfan Foundation and Baylor College of Medicine. The study is sponsored by the National Marfan Foundation, USA, and the Michael E. DeBakey Department of Surgery at Baylor College of Medicine, USA.

Read at the Eighty-eighth Annual Meeting of The American Association for Thoracic Surgery, San Diego, Calif, May 10-14, 2008.

Received for publication May 6, 2008; revisions received Oct 9, 2008; accepted for publication Nov 15, 2008.

Address for reprints: Irina V. Volguina, PhD, Baylor College of Medicine, Division of Cardiothoracic Surgery, One Baylor Plaza, BCM 390, Houston, TX 77030 (E-mail: volguina@bcm.edu).

J Thorac Cardiovasc Surg 2009;137:1124-32

$0022-5223 / \$ 36.00$

Copyright (c) 2009 by The American Association for Thoracic Surgery doi: $10.1016 /$ j.jtcvs.2009.03.023
Patients with Marfan syndrome (MFS) have an inborn connective tissue defect that involves multiple organ systems. ${ }^{1}$ Aortic root dilatation is the predominant cardiovascular lesion, affecting about $64 \%$ of children and $80 \%$ of adults with MFS. If untreated, it can lead to life-threatening aortic dissection, rupture, or both. ${ }^{2-4}$

Replacing the aortic valve and root with a composite valve graft has been the mainstay of surgical treatment of aortic root abnormalities since the technique was introduced by Bentall and de Bono ${ }^{5}$ in the 1960 s. $^{6-9}$ Techniques for aortic valvesparing (AVS) root replacement were subsequently introduced by Yacoub in 1979 (remodeling) and David in 1988 (reimplantation). ${ }^{10,11}$ Concern about potential deterioration of the preserved aortic valve leaflets has created controversy regarding the durability of AVS reconstructions in patients with MFS. ${ }^{12-14}$ Despite this concern, and although AVS root replacement operations are more technically demanding than aortic valve-replacing (AVR) procedures, AVS approaches have been enthusiastically accepted by many surgeons and MFS patients because anticoagulation is not necessary. A prospective, international registry study-Aortic Valve Operative Outcomes in Marfan Patients-was initiated to provide contemporary comparative data on short-term 


\section{Abbreviations and Acronyms \\ AVR $=$ aortic valve-replacing \\ AVS $=$ aortic valve-sparing \\ DCC $=$ Data Coordination Center \\ MFS $=$ Marfan syndrome}

clinical outcomes after AVS and AVR root operations in patients with MFS. The purpose of this initial report is to describe the registry study design and to compare the early outcomes in the first 151 enrolled patients.

\section{PATIENTS AND METHODS}

\section{Overall Study Design, Study Organization, and Patient Recruitment Protocol}

This multicenter study is designed to prospectively evaluate clinical outcomes after aortic root replacement using either AVR or AVS techniques in patients with MFS. There is no attempt to assign the type of repair that patients receive; the choice of operation is based on clinical factors and on surgeon and patient preference. The primary end point for the study is the composite incidence of all valve-related complications. Based on an estimated event rate of $20 \%$, the study is designed with a target sample size of 250 , which provides $80 \%$ power $(\alpha=.05)$ to detect a 1.85 -fold increase in the relative risk of valve-related complications between the AVR and AVS groups.

The Data Coordination Center (DCC), Marfan Diagnostic Core, Imaging Core, Surgical Core, and Genetic Repository coordinate the study. The DCC developed the study protocol and monitors its implementation in terms of data collection and management, as well as providing coordination and administrative support to all participating institutions. The study protocol has been approved by the institutional review board or ethics committee at each participating institution. The Marfan Diagnostic Core reviews each patient's eligibility for enrollment by verifying the clinical diagnosis of MFS. Imaging studies are collected and analyzed by the Imaging Core. The Surgical Core manages the collection of standardized surgical data. Blood and aortic tissue samples are obtained and stored at the study's Genetic Repository for future analysis and will be available to interested investigators.

Patients with a confirmed or tentative diagnosis of MFS who need aortic root surgery are consecutively approached for participation in the study, including patients having emergent or urgent surgery. All recruited patients sign a consent form. To participate, patients must meet strict Ghent criteria, ${ }^{1}$ need aortic root surgery, and be available for follow-up.

\section{Data Collection and Definitions}

The study sites prospectively collect clinical data on preoperative status, operation type and details, and postoperative and follow-up status by using data collection forms developed by the DCC and approved by the Professional Advisory Board of the National Marfan Foundation. Follow-up information is obtained $6,12,24$, and 36 months after surgery. Whenever available, echocardiograms performed before surgery, at discharge, and during follow-up are collected and reviewed by the Imaging Core. Before the operation and 1 year afterward, patients complete the SF36v2 health-related quality of life questionnaire (QualityMetric, Inc, Lincoln, RI). After each operation, the surgeon completes a questionnaire on the timing of the decision between AVR and AVS techniques and the reasons for the surgeon's choice.

All study sites enter their data into a secure on-line data collection and study management system provided and maintained by Velos, Inc (Freemont, Calif). Initial data validation is performed by built-in data type and range/limit checks. Mandatory data entry for selected fields prevents the omission of data for essential variables. During quarterly data reviews, the DCC performs logic checks, identifies all missing data in the nonman- datory fields, and requests clarification of discrepancies and submission of missing data from study sites. Finally, the DCC performs annual visits to selected sites to review study documentation, assure protocol compliance, and check on-line data entries against source documentation for approximately $15 \%$ of enrolled patients.

In the present analysis, operations were categorized as AVS or AVR according to the initial procedure performed. The AVS procedures were described according to the classification system suggested by Miller ${ }^{12}$ in 2003. Emergent operations were defined as mandated within 24 hours after presentation because of the patient's medical condition, urgent operations as mandated within 7 days after presentation, and elective operations as performed at the convenience of both the surgeon and the patient. Duration of surgery was defined as the interval between skin incision and skin closure.

The definitions of valve-related morbidity and mortality were based on the recommendations in the 1996 guidelines written by Edmunds and colleagues. ${ }^{15}$ Valve-related complications were defined as structural valvular deterioration, nonstructural valve dysfunction, valve thrombosis, embolism (ie, neurologic events and peripheral embolic events), operated valvular endocarditis, or bleeding. Bleeding events were considered to be valverelated complications if they occurred after discharge and caused death, hospitalization, or permanent injury or if they necessitated transfusion, regardless of whether the patient was taking anticoagulant or antiplatelet drugs; early postoperative bleeding events, such as mediastinal hemorrhage necessitating re-exploration, were recorded separately and were not categorized as valve-related complications. Cardiac complications encompassed myocardial infarction, atrial or ventricular arrhythmia requiring treatment, pericardial effusion necessitating drainage, and cardiac failure necessitating inotropic support for more than 48 hours, intra-aortic balloon counterpulsation, or mechanical ventricular assistance. Pulmonary complications included the need for more than 48 hours of ventilatory support, respiratory failure necessitating reintubation, adult respiratory distress syndrome, atelectasis necessitating bronchoscopy, chylothorax, prolonged air leak, pneumonia, and pleural effusion or pneumothorax necessitating an evacuation procedure. Acute renal dysfunction was defined as a serum creatinine level that was more than double the baseline level.

\section{Patients and Operations}

Two hundred fourteen consecutive patients who required aortic root replacement and were suspected to have MFS were approached for potential enrollment in the study at 18 tertiary institutions in North and South America and Europe during the period of March 1, 2005, to January 31, 2008. Of these patients, 6 declined to participate, 23 did not meet Ghent criteria, and 22 had not yet completed the diagnostic screening process. Thus, 163 patients met the screening criteria and were enrolled; this group represents $65 \%$ of the 250 patients needed to complete study enrollment. Twelve of the patients had been enrolled recently and did not yet have complete 30-day data; these patients were excluded, leaving 151 patients in the analysis of early outcomes.

Table 1 provides demographic and clinical information obtained at presentation, by type of operation. The types of aortic root replacement procedures and the details of the operations are provided in Tables 2 and 3, respectively. Aortic root replacement was performed through a standard, full median sternotomy in $145(96 \%)$ patients. Of the 6 remaining patients, 4 had transverse sternotomies, and 2 had upper hemisternotomies. One hundred five $(70 \%)$ patients underwent an AVS procedure. The David V technique, used in 57 (54\%) AVS patients, was the most common procedure. All but one of the AVS patients had a reimplantation procedure; one Yacoub remodeling operation was performed. Two AVS patients required intraoperative conversion to AVR; they were included in the AVS group for analysis because AVS was the initial procedure performed. Either direct cusp repair or shortening of the cusp free margin was performed in $22(21 \%)$ patients; of these patients, $15(68 \%)$ had both cusp repair and free margin shortening, 5 $(23 \%)$ had only free margin shortening, and $2(9 \%)$ had only direct cusp repair. Forty-six $(30 \%)$ patients underwent AVR procedures; most of these 
TABLE 1. Presenting characteristics of 151 patients with MFS undergoing aortic root replacement

\section{Variable}

Age, $y$, mean \pm SD (range)

Male sex

Hypertension

Hypercholesterolemia

Diabetes

Current smoker

Former smoker

Recent cerebrovascular accident

Chronic obstructive lung disease

Pneumonia

Pulmonary hypertension

Coronary artery disease

Myocardial infarction within $24 \mathrm{~h}$

Cardiomyopathy

Mitral valve disease

Endocarditis

Coagulopathy

NYHA class

I

II

III

IV

Left ventricular ejection fraction, $\%$, median (IQR) (No. of observations)

Sinus rhythm

Aortic regurgitation (No. of observations)

None/trivial

Mild

Moderate

Severe

Aortic dimensions, mm

Annulus, mean \pm SD (No. of observations)

Sinuses of Valsalva, median (IQR)

(No. of observations)

Sinotubular junction, mean \pm SD

(No. of observations)

Ascending aortic dissection

Acute dissection

Chronic dissection

Aortic rupture

Previous CV surgery

No. of previous CV operations

One

Two

Three

Type of previous CV surgery

Isolated aortic valve replacement

Homograft root

Mitral valve repair/replacement

Aortic aneurysm repair

Ascending aorta

Descending thoracic aorta

Abdominal aorta

Total $(\mathbf{n}=\mathbf{1 5 1})$

$33 \pm 13(4-69)$

$102(68 \%)$

$37(25 \%)$

$6(4 \%)$

$1(1 \%)$

$17(12 \%)$

$42(29 \%)$

$4(3 \%)$

$1(1 \%)$

$1(1 \%)$

$2(1 \%)$

$1(1 \%)$

$1(1 \%)$

$2(1 \%)$

$35(23 \%)$

0

$4(3 \%)$

$109(74 \%)$

$30(20 \%)$

$6(4 \%)$

$3(2 \%)$

60 (55-65)

(139)

147 (97\%)

(144)

$95(66 \%)$

$26(18 \%)$

$9(6 \%)$

$14(10 \%)$
$26 \pm 3(114)$
50 (47-54)
(124)

$38 \pm 8(108)$

$12(8 \%)$

$6(4 \%)$

$6(4 \%)$

0

$10(7 \%)$

$8(5 \%)$

$1(1 \%)$

$1(1 \%)$

$1(1 \%)$

$1(1 \%)$

$2(1 \%)$

$1(1 \%)$

$1(1 \%)$

$1(1 \%)$

\begin{abstract}
$\operatorname{AVR}(n=46)$
\end{abstract}

$39 \pm 13(16-69)$

$34(74 \%)$

$13(28 \%)$

$2(4 \%)$

0

$5(11 \%)$

$11(25 \%)$

$3(7 \%)$

0

$1(2 \%)$

0

0

0

$1(2 \%)$

$10(22 \%)$

0

$2(4 \%)$

$32(73 \%)$

$8(18 \%)$

$3(7 \%)$

$1(2 \%)$

60 (55-65)

(45)

$45(98 \%)$

(45)

$24(53 \%)$

$6(13 \%)$

$6(13 \%)$

$9(20 \%)$

$$
\begin{aligned}
& 27 \pm 3(39) \\
& 52(50-56)
\end{aligned}
$$

(41)

$39 \pm 10(37)$

$6(13 \%)$

$3(7 \%)$

$3(7 \%)$

0

$6(13 \%)$

$5(11 \%)$

0

$1(2 \%)$

$1(2 \%)$

$1(2 \%)$

0

$1(2 \%)$

0

$1(2 \%)$
$\operatorname{AVS}(\mathbf{n}=\mathbf{1 0 5})$

$31 \pm 12(4-64)$

$68(65 \%)$

$24(23 \%)$

$4(4 \%)$

$1(1 \%)$

$12(12 \%)$

$31(30 \%)$

$1(1 \%)$

$1(1 \%)$

0

$2(2 \%)$

$1(1 \%)$

$1(1 \%)$

$1(1 \%)$

$25(24 \%)$

0

$2(2 \%)$

$77(74 \%)$

$22(21 \%)$

$3(3 \%)$

$2(2 \%)$

60 (55-65)

(94)

$102(97 \%)$

(99)

$71(72 \%)$

$20(20 \%)$

$3(3 \%)$

$5(5 \%)$

$26 \pm 3(75)$

$50(47-52)$

(83)

$38 \pm 7$ (71)

$6(6 \%)$

$3(3 \%)$

$3(3 \%)$

0

$4(4 \%)$

$3(3 \%)$

$1(1 \%)$

0

0

0

$2(2 \%)$

0

$1(1 \%)$

0
$.6 \dagger$

$1.0^{*}$

$<.01$

$P$ value

$<.01$

.3

.5

$1.0^{*}$

$1.0 *$

.8

$.08 *$

$1.0 *$

$.3 *$

$1.0 *$

$1.0 *$

$1.0^{*}$

$.5 *$

.8

-

.6*

.7

$6 \dagger$

列

.1

.2

.4*

$.4 *$

-

$.07 *$

$.1 *$

$1.0 *$

$.3 *$

$.3 *$

$.3 *$

$1.0 *$

$.3 *$

$1.0 *$

$.3^{*}$ 
TABLE 1. Continued

\begin{tabular}{|c|c|c|c|c|}
\hline Variable & Total $(n=151)$ & $\operatorname{AVR}(n=46)$ & $\operatorname{AVS}(n=105)$ & $P$ value \\
\hline \multicolumn{5}{|l|}{ Aortic dissection repair } \\
\hline Stanford A & $3(2 \%)$ & $3(7 \%)$ & 0 & $.03 *$ \\
\hline Stanford B & $2(1 \%)$ & 0 & $2(2 \%)$ & $1.0 *$ \\
\hline Other CV operations & $6(4 \%)$ & $3(7 \%)$ & $3(3 \%)$ & $.4 *$ \\
\hline Creatinine, $\mathrm{mg} / \mathrm{dL}$, mean $\pm \mathrm{SD}$ & $0.9 \pm 0.2$ & $1.0 \pm 0.2$ & $0.9 \pm 0.2$ & $<.01$ \\
\hline Renal failure & $1(1 \%)$ & $1(2 \%)$ & 0 & $.3 *$ \\
\hline
\end{tabular}

Data are presented as number and percentage of patients unless otherwise specified. $M F S$, Marfan syndrome; $A V R$, aortic valve-replacing; $A V S$, aortic valve-sparing; $S D$, standard deviation; NYHA, New York Heart Association; $I Q R$, interquartile range; $C V$, cardiovascular. *Fisher's exact test. $\dagger$ Kolmogorov-Smirnov 2 -sample test.

patients $(\mathrm{n}=39 ; 85 \%)$ received a composite valve graft incorporating a mechanical prosthesis.

\section{Follow-up Data}

Thirty-day and hospital outcome data were complete for all 151 patients. Discharge echocardiograms were obtained for $144(95 \%)$ patients. In 100 $(69 \%)$ of the echocardiograms, the Imaging Core was able to assess aortic valve function. Two echocardiograms did not provide adequate visualization of the aortic valve. For the remaining 42 patients, Imaging Core analysis was not possible because of logistical or data formatting problems; for these patients, data were abstracted from the study-site echocardiogram reports.

\section{Statistical Analysis}

Given the distribution of AVR and AVS procedures in the first $151 \mathrm{pa}-$ tients, this analysis had $80 \%$ power to detect a relative risk of $2.15(\alpha=.05)$ for valve-related complications, the primary study end point. Normally distributed continuous variables were presented as mean \pm SD and compared between groups by Student $t$ tests. Nonnormally distributed continuous variables were presented as medians and interquartile ranges; the KolmogorovSmirnov test was used for between-group comparisons. Categorical variables were presented as frequencies and percentages; $\chi^{2}$ or Fisher's exact tests were used for between-group comparisons, as appropriate.

To control for confounding factors, we performed stepwise multivariable logistic regression analysis to determine whether type of operative procedure was an independent predictor of early valve-related complications. Univariate predictors with $P$ values less than .2 were included in the regression model. Data on 140 patients were complete and therefore included in the model. The DCC performed the statistical analyses with SPSS 16.0 statistical software (SPSS Inc, Chicago, Ill).

TABLE 2. Types of aortic root replacement operations in 151 patients with MFS

\begin{tabular}{lc}
\hline \multicolumn{1}{c}{ Type of root replacement } & No. $(\mathbf{n}=\mathbf{1 5 1})$ \\
\hline Valve-replacing & $46(30 \%)$ \\
Mechanical composite valve graft & 39 \\
Stented bioprosthetic composite valve graft & 5 \\
Homograft & 1 \\
Stentless porcine root & 1 \\
Valve-sparing & $105(70 \%)$ \\
Reimplantation & 104 \\
David V & 57 \\
David I & 38 \\
David IV & 8 \\
Florida sleeve & 1 \\
Remodeling & 1 \\
\hline$M F S$, Marfan syndrome. &
\end{tabular}

\section{RESULTS \\ Overall Outcomes}

No 30-day or in-hospital deaths occurred in either group. There were no strokes and no reoperations on the aortic valve. One patient undergoing AVS surgery required reoperation to correct coronary artery kinking after a Florida sleeve procedure. In 2 patients, heart block developed that necessitated placement of a permanent pacemaker. The overall incidence of valve-related complications at 30 days was $3 \%$ (Table 4); the complications included 1 bleeding event, 1 reversible ischemic neurologic deficit, and 3 cases of intraoperative nonstructural valve dysfunction. The valve-related bleeding event was a bloody pericardial effusion that occurred after discharge on the 15 th postoperative day and required pericardial drainage. All other early bleeding events occurred before discharge and did not meet the criteria for classification as valve-related complications. Thirteen $(9 \%)$ patients required re-exploration for bleeding; a bleeding site involving the root repair (eg, at the annulus or a coronary anastomosis) was identified in 3 of these patients. Five patients with bleeding did not require mediastinal re-exploration. Of those, 3 had bloody pericardial effusions with cardiac tamponade ( 2 were drained, and 1 was treated nonoperatively), 1 had early postoperative bleeding that did not require reoperation, and 1 had a right hemothorax that required drainage. Two patients having AVS surgery had embolic complications: 1 patient had a reversible ischemic neurologic deficit 7 days after surgery, and 1 had a pulmonary embolus on postoperative day 12; the latter was not considered valve-related. Three patients had nonstructural valve dysfunction resulting in aortic regurgitation that was detected intraoperatively and treated during the same operation. Two of these patients had unsuccessful attempts at AVS root replacement (1 Yacoub remodeling and 1 David I reimplantation) and required conversion to AVR. The third patient with nonstructural valve dysfunction had substantial aortic regurgitation immediately after a cryopreserved homograft root was implanted; the homograft valve leaflets were excised and replaced with a stented bioprosthetic valve. There were no early cases of structural valve deterioration, valve thrombosis, or endocarditis. Of the 144 patients with postoperative echocardiograms, aortic valve function 
TABLE 3. Operative details for 151 aortic root replacements in patients with MFS

\begin{tabular}{|c|c|c|c|c|}
\hline Variable & Total $(n=151)$ & $\operatorname{AVR}(n=46)$ & $\operatorname{AVS}(n=105)$ & $P$ value \\
\hline \multicolumn{5}{|l|}{ Urgency of surgery } \\
\hline Elective & $134(89 \%)$ & $35(76 \%)$ & $99(94 \%)$ & $<.01$ \\
\hline Urgent & $6(4 \%)$ & $3(7 \%)$ & $3(3 \%)$ & \\
\hline Emergent & $11(7 \%)$ & $8(17 \%)$ & $3(3 \%)$ & \\
\hline \multicolumn{5}{|l|}{ Perfusion technique } \\
\hline Cardiopulmonary bypass alone & $109(72 \%)$ & $33(72 \%)$ & $76(72 \%)$ & .9 \\
\hline Circulatory arrest & $42(28 \%)$ & $13(28 \%)$ & $29(28 \%)$ & .9 \\
\hline HCA without perfusion adjuncts & $13(9 \%)$ & $3(7 \%)$ & $10(10 \%)$ & $.8^{*}$ \\
\hline HCA with ACP & $10(7 \%)$ & $3(7 \%)$ & $7(7 \%)$ & $1.0^{*}$ \\
\hline HCA with RCP & $16(11 \%)$ & $6(13 \%)$ & $10(10 \%)$ & .5 \\
\hline HCA with $\mathrm{ACP}+\mathrm{RCP}$ & $3(2 \%)$ & $1(2 \%)$ & $2(2 \%)$ & $1.0^{*}$ \\
\hline Cardiopulmonary bypass time, min, median (IQR) & $177(142-252)$ & $148(107-199)$ & $191(157-271)$ & $<.01 \dagger$ \\
\hline Aortic crossclamp time, min, median (IQR) & $140(108-208)$ & $114(76-166)$ & $150(120-225)$ & $<.01 \dagger$ \\
\hline \multicolumn{5}{|l|}{ Concomitant procedures } \\
\hline Hemiarch & $31(21 \%)$ & $10(22 \%)$ & $21(20 \%)$ & .8 \\
\hline Full arch (without elephant trunk) & $4(3 \%)$ & 0 & $4(4 \%)$ & $.3^{*}$ \\
\hline Elephant trunk arch & $4(3 \%)$ & $3(7 \%)$ & $1(1 \%)$ & $.08^{*}$ \\
\hline Mitral valve replacement & $2(1 \%)$ & $1(2 \%)$ & $1(1 \%)$ & $.5^{*}$ \\
\hline Mitral valve repair & $10(7 \%)$ & $2(4 \%)$ & $8(8 \%)$ & $.5^{*}$ \\
\hline Coronary artery bypass & $6(4 \%)$ & $3(7 \%)$ & $3(3 \%)$ & $.4^{*}$ \\
\hline Duration of surgery, min, median (IQR) & $335(259-395)$ & $293(230-367)$ & $340(274-420)$ & $.02 \dagger$ \\
\hline
\end{tabular}

Data are presented as number and percentage of patients unless otherwise specified. $M F S$, Marfan syndrome; $A V R$, aortic valve-replacing; $A V S$, aortic valve-sparing; $H C A$, hypothermic circulatory arrest; $A C P$, antegrade cerebral perfusion; $R C P$, retrograde cerebral perfusion; $I Q R$, interquartile range. *Fisher's exact test; $\dagger$ Kolmogorov-Smirnov 2 -sample test.

could be assessed in 142; 140 patients had no or trivial aortic valve regurgitation, and 2 had mild regurgitation.

\section{Comparison of AVR and AVS Groups}

Compared with patients who underwent AVR root replacement, those who underwent AVS procedures were significantly younger and were less likely to have had previous repair of ascending aortic dissection (Table 1). There were trends toward fewer recent cerebrovascular accidents and fewer previous cardiovascular operations in the AVS group. Severe aortic regurgitation was more common in the AVR group $(9 / 45,20 \%)$ than in the AVS group (5/99, 5\%; $P=.005)$. Preoperative New York Heart Association class, aortic root size, left ventricular ejection fraction, and smoking history were not significantly different between the two groups.

Questionnaire data were obtained from operating surgeons (on the timing of their decision between AVR and AVS techniques and the reasons for their choice) for all patients in the AVR group and for $101(96 \%)$ patients in the AVS group. The final decision whether to perform an AVR or AVS procedure was made intraoperatively for $65(43 \%)$ of the patients. For 32 patients $(21 \%$ overall and $70 \%$ of the AVR group), AVR was considered the only option because the condition of the aortic valve or root was deemed unsuitable for an AVS procedure. Twelve $(26 \%)$ of the AVR patients could have had an AVS procedure but chose AVR instead. Three AVR patients were already on a regi- men of warfarin therapy for other reasons. In the AVS group, $78(77 \%)$ patients chose this type of operation because they wanted to avoid anticoagulation; 19 of these patients were women of childbearing age. Seven $(7 \%)$ patients followed their surgeon's preference for AVS surgery. Sixteen $(16 \%)$ patients requested AVS, but did not specify the reason for selecting this procedure.

The AVR group had a significantly higher proportion of patients who underwent emergent and urgent operations (Table 3). The AVS group had longer operative times, including total duration of surgery, aortic clamp time, and cardiopulmonary bypass time. Similar proportions of AVR and AVS patients underwent concomitant surgical procedures.

Two (4\%) of the 30-day valve-related complications occurred in the AVR group, and $3(3 \%)$ occurred in the AVS group; the difference did not reach statistical significance $(P=.6)$. Logistic regression analysis did not identify the type of operative procedure as an independent predictor of 30-day valve-related complications $(P=.5)$. There were no significant differences in the rates of cardiac, pulmonary, and renal complications (Table 4). The total postoperative ventilatory support time, total time in the intensive care unit, and length of hospital stay did not differ significantly between the AVR and AVS groups. At discharge, cardiac rhythm status, New York Heart Association class, and left ventricular ejection fraction were similar (Table 5). 
TABLE 4. Early outcomes after aortic root replacement in 151 patients with MFS

\begin{tabular}{|c|c|c|c|c|}
\hline Outcomes & Total $(\mathbf{n}=\mathbf{1 5 1})$ & $\operatorname{AVR}(n=46)$ & $\operatorname{AVS}(\mathbf{n}=\mathbf{1 0 5})$ & $P$ value \\
\hline Thirty-day valve-related complications & $5(3 \%)$ & $2(4 \%)$ & $3(3 \%)$ & $.6^{*}$ \\
\hline Structural valvular deterioration & 0 & 0 & 0 & - \\
\hline Nonstructural dysfunction & $3(2 \%)$ & $1(2 \%)$ & $2(2 \%)$ & $.5^{*}$ \\
\hline Valve thrombosis & 0 & 0 & 0 & - \\
\hline Embolism & $1(1 \%)$ & 0 & $1(1 \%)$ & $1.0^{*}$ \\
\hline Bleeding & $1(1 \%)$ & $1(2 \%)$ & 0 & $.3^{*}$ \\
\hline Operated valvular endocarditis & 0 & 0 & 0 & - \\
\hline Mediastinal re-exploration & $14(9 \%)$ & $6(13 \%)$ & $8(8 \%)$ & $.4^{*}$ \\
\hline Cardiac complications & $31(21 \%)$ & $12(26 \%)$ & $19(18 \%)$ & .3 \\
\hline $\begin{array}{l}\text { Atrial or ventricular arrhythmia requiring } \\
\text { treatment }\end{array}$ & $23(15 \%)$ & $9(20 \%)$ & $14(13 \%)$ & .3 \\
\hline Pericardial effusion requiring drainage & $5(3 \%)$ & $2(4 \%)$ & $3(3 \%)$ & $.6^{*}$ \\
\hline Cardiac failure & $4(3 \%)$ & $1(2 \%)$ & $3(3 \%)$ & $1.0^{*}$ \\
\hline Pulmonary complications & $11(7 \%)$ & $6(13 \%)$ & $5(5 \%)$ & $.09 *$ \\
\hline $\begin{array}{l}\text { Total postoperative ventilation support time, } \\
\text { h, median (IQR) }\end{array}$ & $11(6-19)$ & $12(6-24)$ & $10(6-18)$ & $.5 \dagger$ \\
\hline Acute renal dysfunction & $1(1 \%)$ & $1(2 \%)$ & 0 & $.3 *$ \\
\hline Multiple organ failure & $1(1 \%)$ & $1(2 \%)$ & 0 & $.3^{*}$ \\
\hline Total time in ICU, h, median (IQR) & $36(24-57)$ & $44(23-67)$ & $32(24-51)$ & $.5 \dagger$ \\
\hline Length of hospital stay, d, median (IQR) & $7(5-11)$ & $7(6-10)$ & $7(5-11)$ & $.8 \dagger$ \\
\hline
\end{tabular}

\section{DISCUSSION}

This study is the first international, multicenter effort to prospectively compare aortic valve operative outcomes in patients with MFS. The multicenter design is advantageous for accumulating data on patients with a rare disease because it allows quicker collection of a sample of adequate size while avoiding the confounding effects of significant changes in surgical technique that may occur over time. It does, of course, make standardization of methods and tech- niques impossible; however, the variability in surgical technique in this series parallels that found in general, which enhances the overall applicability of the results derived from this investigation.

By design, this registry study does not involve random assignment of root replacement techniques. A nonrandomized approach is appropriate for studies comparing these two procedures, as suggested by our finding that the final decision about which technique to use was made intraoperatively in

TABLE 5. Cardiac status at the time of discharge

\begin{tabular}{lccc}
\hline \multicolumn{1}{c}{ Variable } & Total $(\mathbf{n}=\mathbf{1 5 1})$ & AVR $(\mathbf{n}=\mathbf{4 6})$ & AVS $(\mathbf{n}=\mathbf{1 0 5})$ \\
\hline Cardiac rhythm & & & $\boldsymbol{P}$ value \\
$\quad$ Sinus & $144(95 \%)$ & $42(91 \%)$ & $102(97 \%)$ \\
Atrial fibrillation/flutter & $3(2 \%)$ & $1(2 \%)$ & $2(2 \%)$ \\
Heart block & $2(1 \%)$ & $2(4 \%)$ & 0 \\
Paced & $2(1 \%)$ & $1(2 \%)$ & $1(1 \%)$ \\
NYHA class & & & $69(66 \%)$ \\
I & $97(65 \%)$ & $28(62 \%)$ & $33(32 \%)$ \\
II & $46(31 \%)$ & $13(29 \%)$ & $2(2 \%)$ \\
III & $4(3 \%)$ & $2(4 \%)$ & 0 \\
IV & $2(1 \%)$ & $2(4 \%)$ & $60(55-65)$ \\
Left ventricular ejection fraction, $\%$, median (IQR) & $60(55-65)$ & $60(55-65)$ & $(95)$ \\
(No. of observations) & $(135)$ & $(40)$ & $(101)$ \\
Aortic regurgitation (No. of observations) & $(142)$ & $(41)$ & $100(99 \%)$ \\
None/trivial & $140(99 \%)$ & $40(98 \%)$ & $1(1 \%)$ \\
Mild & $2(1 \%)$ & $1(2 \%)$ & 0 \\
Moderate & 0 & 0 & $.5 \%$ \\
Severe & 0 & 0 & 0 \\
\hline
\end{tabular}

Data are presented as number and percentage of patients unless otherwise specified. $A V R$, aortic valve-replacing; $A V S$, aortic valve-sparing; $N Y H A$, New York Heart Association; $I Q R$, interquartile range. *Fisher's exact test; $†$ Kolmogorov-Smirnov 2-sample test. 
more than $40 \%$ of cases. A randomized trial would require extremely narrow anatomic inclusion criteria to minimize the crossover rate, making it difficult to enroll a sufficient number of patients in a realistic time frame. Although the nonrandomized design will make it impossible to determine whether one method is superior to the other, the data from this prospective study provide the most accurate comparison of these surgical techniques to date.

The analysis of early outcomes showed that AVS was performed in $70 \%$ of patients with MFS undergoing aortic root replacement, which is consistent with previously published results: $73 \%$ of 112 consecutive MFS patients in a series published by Birks and colleagues ${ }^{16}$ underwent AVS surgery, as well as $58 \%$ of 105 patients in a series by de Oliveira and colleagues ${ }^{17}$ and $60 \%$ of 144 patients in a recently published study by Patel and colleagues. ${ }^{18}$

Of note, the Yacoub remodeling technique was used in only 1 of the AVS patients in the current series; this result reflects the substantial reduction in recent years of surgeons' enthusiasm for using remodeling techniques in patients with MFS, at least in these 18 centers. Birks and colleagues ${ }^{16}$ endorsed the remodeling procedure in a retrospective analysis of 82 MFS patients in 1999; the results were marred, however, by relatively high rates of reoperation and aortic regurgitation within 5 to 10 years. Since that time, several groups have expressed concerns that remodeling carries a higher risk for complications-including operative bleeding, progressive annular dilatation, and aortic regurgitation-than does the reimplantation technique. ${ }^{12,14,17,19-23}$

The absence of early deaths after 151 root replacement operations in this multicenter study is extremely encouraging; $100 \%$ early survival - which was also recently reported for 59 MFS patients by Kallenbach and colleagues, ${ }^{24}$ for 35 patients by Settepani and colleagues, ${ }^{25}$ and for 140 patients by Patel and colleagues ${ }^{18}$-represents an improvement over survival rates in earlier series. ${ }^{6,7,16,17}$ At this stage of the study, the type of aortic root replacement does not appear to be a predictor of early valve-related complications. We recognize, however, that no long-term inferences should be drawn from these preliminary results and that further follow-up data accumulation and analysis are necessary.

The limitations of this registry study include the inherent drawbacks of its nonrandomized design, such as the potential for confounding by clinical indication bias, the inability to adjust for unknown confounding factors, and the difference in the sizes of the comparison groups; the latter was not anticipated at the beginning of the study and may incur power limitations. Additionally, retrieval and core laboratory analysis of all original echocardiographic images have been particularly challenging. European sites do not routinely perform postoperative echocardiograms for AVR patients, and, in several instances, the images from international sites had image formatting compatibility issues. Finally, it is important to recognize that this study was not intended to evaluate differences in long-term durability, which is certainly an end point of major clinical importance; at least 10 years of follow-up would be required to address this issue.

To summarize, the analysis of early outcomes revealed that AVS was more common than AVR in MFS patients undergoing root replacement at the participating centers. Although AVS procedures required longer aortic clamp and cardiopulmonary bypass times, the added complexity of AVS root replacement did not translate into adverse early outcomes. Subsequent analyses will compare the 3-year durability of these two approaches.

Stephen N. Palmer, PhD, ELS, contributed to the editing of this manuscript.

\section{References}

1. De Paepe A, Devereux RB, Dietz HC, Hennekam RC, Pyeritz RE. Revised diagnostic criteria for the Marfan syndrome. Am J Med Genet. 1996;62:417-26.

2. Coselli JS, LeMaire SA. Aortic manifestations and surgery in Marfan syndrome in pediatric patients. Prog Pediatr Cardiol. 1996;5:189-203.

3. Roman MJ, Rosen SE, Kramer-Fox R, Devereux RB. Prognostic significance of the pattern of aortic root dilation in the Marfan syndrome. J Am Coll Cardiol. 1993;22:1470-6.

4. Tsipouras P, Silverman DI. The genetic basis of aortic disease: Marfan syndrome and beyond. Cardiol Clin. 1999;17:683-96.

5. Bentall H, De Bono A. A technique for complete replacement of the ascending aorta. Thorax. 1968;23:338-9.

6. Gott VL, Cameron DE, Alejo DE, Greene PS, Shake JG, Caparrelli DJ, et al. Aortic root replacement in 271 Marfan patients: a 24-year experience. Ann Thorac Surg. 2002;73:438-43

7. Gott VL, Greene PS, Alejo DE, Cameron DE, Naftel DC, Miller DC, et al. Replacement of the aortic root in patients with Marfan's syndrome. N Engl J Med. 1999;340:1307-13.

8. Kouchoukos NT, Karp RB, Lell WA. Replacement of the ascending aorta and aortic valve with a composite graft: results in 25 patients. Ann Thorac Surg. 1977;24: $140-8$.

9. LeMaire SA, Carter SA, Volguina IV, Laux AT, Milewicz DM, Borsato GW, et al. Spectrum of aortic operations in 300 patients with confirmed or suspected Marfan syndrome. Ann Thorac Surg. 2006;81:2063-78.

10. Fagan A, Pillai R, Radley-Smith R, Yacoub MH. Results of new valve conserving operation for treatment of aneurysms or acute dissection of the aortic root. $\mathrm{Br}$ Heart J. 1983;49:302.

11. David TE, Feindel CM. An aortic valve-sparing operation for patients with aortic incompetence and aneurysm of the ascending aorta. J Thorac Cardiovasc Surg. 1992;103:617-21.

12. Miller DC. Valve-sparing aortic root replacement in patients with the Marfan syndrome. J Thorac Cardiovasc Surg. 2003;125:773-8.

13. Cameron DE, Vricella LA. Valve-sparing aortic root replacement in Marfan syndrome. Semin Thorac Cardiovasc Surg Pediatr Card Surg Annu. 2005;103-11.

14. Miller DC. Valve-sparing aortic root replacement: current state of the art and where are we headed? Ann Thorac Surg. 2007;83:S736-9.

15. Edmunds LH Jr, Clark RE, Cohn LH, Grunkemeier GL, Miller DC, Weisel RD. Guidelines for reporting morbidity and mortality after cardiac valvular operations. Ad Hoc Liaison Committee for Standardizing Definitions of Prosthetic Heart Valve Morbidity of The American Association for Thoracic Surgery and The Society of Thoracic Surgeons. J Thorac Cardiovasc Surg. 1996;112:708-11.

16. Birks EJ, Webb C, Child A, Radley-Smith R, Yacoub MH. Early and long-term results of a valve-sparing operation for Marfan syndrome. Circulation. 1999; 100(19 Suppl):II29-35.

17. de Oliveira NC, David TE, Ivanov J, Armstrong S, Eriksson MJ, Rakowski H, et al. Results of surgery for aortic root aneurysm in patients with Marfan syndrome. J Thorac Cardiovasc Surg. 2003;125:789-96.

18. Patel ND, Weiss ES, Alejo DE, Nwakanma LU, Williams JA, Dietz HC, et al. Aortic root operations for Marfan syndrome: a comparison of the Bentall and valve-sparing procedures. Ann Thorac Surg. 2008;85:2003-11. 
19. Cattaneo SM, Bethea BT, Alejo DE, Spevak PJ, Clauss SB, Dietz HC, et al. Surgery for aortic root aneurysm in children: a 21 -year experience in 50 patients. Ann Thorac Surg. 2004;77:168-76.

20. David TE. Reoperations on the aortic valve combined with replacement of the ascending aorta. J Card Surg. 2002;17:46-50.

21. David TE, Armstrong S, Ivanov J, Feindel CM, Omran A, Webb G. Results of aortic valve-sparing operations. J Thorac Cardiovasc Surg. 2001;122: $39-46$.

22. David TE, Feindel CM, Webb GD, Colman JM, Armstrong S, Maganti M. Longterm results of aortic valve-sparing operations for aortic root aneurysm. J Thorac Cardiovasc Surg. 2006;132:347-54.

\section{Appendix}

\section{Study Cores: Institution (Core Director)}

Data Coordination Center and Surgical Core: Baylor College of Medicine (J. S. Coselli, Study Principal Investigator)

Marfan Diagnostic Core: Johns Hopkins Hospital (H.

C. Dietz)

Imaging Core: Mayo Clinic, Rochester (H. M. Connolly) Genetic Repository: University of Texas Medical School at Houston (D. M. Milewicz)

Participating Study Sites: Institution (Site Principal Investigator, Number of Patients Enrolled)

Argentina: Institute of Cardiology and Cardiovascular Surgery-Favaloro Foundation (R. R. Favaloro, 10 patients).

Canada: University of Ottawa Heart Institute (K-L. Chan, 1 patient).

Germany: Hannover Medical School (A. Haverich, 5 patients); University Clinic of Schleswig-Holstein (H. H. Sievers, 6 patients); University of Leipzig (F. W. Mohr, 14 patients).

Netherlands: Leiden University Medical Center (M. I. M. Versteegh, 1 patient).

USA: Baylor College of Medicine (J. S. Coselli, 8 patients); Central Maine Heart and Vascular Institute (R. P. Cochran, C. Frumiento, 1 patient); Johns Hopkins Hospital (V. L. Gott, L. A. Vricella, 16 patients); Loyola University Medical Center (J. P. Schwartz, 3 patients); Mayo Clinic, Rochester (T. M. Sundt III, 21 patients); Missouri Baptist Medical Center (N. T. Kouchoukos, 5 patients); Montefiore Medical Center (A. DeAnda, 2 patients); New York PresbyterianCornell Hospital (L. N. Girardi, 7 patients); Northwestern University Feinberg School of Medicine (T. G. Gleason, C. Malaisrie, 2 patients); Stanford University (D. C. Miller, 19 patients); University of Pennsylvania (J. E. Bavaria, 19 patients); Washington University (M. R. Moon, 11 patients).

\section{Discussion}

Dr Alan D. Hilgenberg (Boston, Mass). Dr Coselli, congratulations to you and your colleagues for designing and implementing this very important study. The presentation is a report of the current practice of aortic root repair in patients with MFS from centers in Europe
23. Svensson LG, Deglurkar I, Ung J, Pettersson G, Gillinov AM, D'Agostino RS, et al. Aortic valve repair and root preservation by remodeling, reimplantation, and tailoring: technical aspects and early outcome. $J$ Card Surg. 2007;22:473-9.

24. Kallenbach K, Baraki H, Khaladj N, Kamiya H, Hagl C, Haverich A, et al. Aortic valve-sparing operation in Marfan syndrome: what do we know after a decade? Ann Thorac Surg. 2007;83:S764-8.

25. Settepani F, Szeto WY, Pacini D, De Paulis R, Chiariello L, Di Bartolomeo R et al. Reimplantation valve-sparing aortic root replacement in Marfan syndrome using the Valsalva conduit: an intercontinental multicenter study. Ann Thorac Surg. 2007;83:S769-73. and North and South America. First of all, it is remarkable that there was no 30-day mortality in 151 patients, attesting to the safety of these complex surgical procedures. Seventy percent of the operations were valve-sparing, all but two of them were of the reimplantation type, and about two thirds included repair with sinuses.

The patients undergoing AVR were different from those undergoing AVS in several important characteristics. They were older, they had more preoperative aortic regurgitation, and they underwent more nonelective operations. These factors probably will affect the long-term results in addition to the presence of a mechanical valve when comparisons with the outcomes of AVS patients are made.

The most important information from this study will come from the years of follow-up data that we hope you will accumulate. The late results of composite valve graft replacement in patients with MFS have been reported at least a couple of times in the last several years. Survival at 10 years of $75 \%$ was reported by Gott in the New England Journal of Medicine study in 1999. More recent data from Hopkins presented at the Society of Thoracic Surgeons meeting in 2008 by Cameron had an $85 \%$ survival at 10 years. Although these results are good, I suspect that the late results in terms of survival and freedom from thromboembolism in the AVS group will be even better in this study. However, how durable will the AVS operations be in terms of avoiding late operation for aortic regurgitation? David and Feindel reported freedom from moderate or severe aortic insufficiency in reimplantation procedures to be $94 \%$ at 10 years, and in their studies, MFS was not a risk factor for late aortic insufficiency. This study should be able to show whether other surgeons throughout the world can achieve similar results. I hope that you will keep gathering this important follow-up data.

I have a couple of questions. Have the participating centers agreed to include their entire experience with consecutive MFS patients, and if not, do you think this will make a difference in the outcomes?

Dr Coselli. They have agreed, and I do believe that not enrolling consecutive patients would affect the results. The intent has been that once investigators agreed to participate in the study, they would recruit and screen consecutive patients. I believe that, so far, compliance has been very good and consecutive patients have been screened.

Dr Hilgenberg. I think that would be important. I am assuming that AVS repair is the preferred operation in all of the centers. What are the common reasons that valves were replaced, if you know, and were there situations in which valves might have been spared if the patient had been referred earlier for surgery? 
Dr Coselli. Those are good questions. In $43 \%$ of the patients, the decision as to which operation to perform was ultimately made at the time of surgery; the decision to replace the valve was made by the surgeon, and it primarily revolved around anatomic features that the surgeons, who are all well-versed in valve-sparing techniques, believed would compromise the durability of a valve-sparing repair.

There were also some patients whose primary concern was that they did not want to have a second procedure and did not want to subject themselves to the risks of the potential lack of durability of the procedure; these patients selected having a mechanical valve at the outset.

Dr Hilgenberg. David and Feindel perform cusp repair frequently in these operations, and I think it would be of interest to the audience to know whether cusp repair was used often or rarely in this series of patients.

Dr Coselli. In this series of patients, relatively rarely; only $17 \%$ of the patients had cusp repair.

Dr Hilgenberg. One final comment: I am sitting here with my colleague, Cary Akins, who formulates the reporting of valve-related complications, and I think the re-exploration for bleeding in this series really does not belong in the valve-related complications. That is a surgical complication, not necessarily valve-related, I believe.

Dr Tirone E. David (Toronto, Ontario, Canada). I am sorry that I am not participating in this study. I think it is important to have information regarding the aortic cusps in this database. In my experience with AVS procedures during the past 19 years, what determines whether a valve can be spared or not is the quality of the aortic cusps. In at least half of my patients with MFS, I had to repair the cusps to spare the valve. In other words, these patients had premature cusp degeneration just as in mitral valve prolapse. Unless you do something with the cusps, I am surprised that you ended up with no aortic insufficiency. Do you have data on how many cusps were abnormal?

Dr Coselli. In the way you describe it, no.

Dr David. I propose that you add this to the database. We find it the single most important determinant of feasibility of repairing these valves. Cusps with large stress fenestrations, overstretched and thinned out, should not be preserved, and AVR probably results in better outcomes.

Dr Coselli. Dr David, thank you for your comments and thank you for your immense contribution and pioneering work in this area. All of the centers that participated involved surgeons who are well-versed in the techniques and carry on a practice that allows them to very reliably carry out AVS procedures; I believe that the results, with no deaths, no strokes, and no moderate or severe aortic valve regurgitation in the early postoperative period, confirm their levels of expertise. I think it would be challenging for this type of registry - which already collects an immense amount of data from very diverse institutions across the globe - to accumulate the kind of detailed information on the leaflets that a single surgeon and a single center can. But I appreciate the insight, and ideally we can incorporate that. 